\title{
Review Article \\ The Role of Hypothalamic Neuropeptides in Neurogenesis and Neuritogenesis
}

\author{
Jan Bakos, ${ }^{1,2}$ Martina Zatkova, ${ }^{1,2}$ Zuzana Bacova, ${ }^{1,3}$ and Daniela Ostatnikova ${ }^{2}$ \\ ${ }^{1}$ Institute of Experimental Endocrinology, Slovak Academy of Sciences, Vlarska 3, 83306 Bratislava, Slovakia \\ ${ }^{2}$ Institute of Physiology, Faculty of Medicine, Comenius University, Sasinkova 2, 81372 Bratislava, Slovakia \\ ${ }^{3}$ Department of Normal and Pathological Physiology, Faculty of Medicine, Slovak Medical University, Limbova 12, \\ 83303 Bratislava, Slovakia \\ Correspondence should be addressed to Jan Bakos; j.bakos@savba.sk
}

Received 20 August 2015; Revised 18 November 2015; Accepted 22 November 2015

Academic Editor: Etienne de Villers-Sidani

Copyright (c) 2016 Jan Bakos et al. This is an open access article distributed under the Creative Commons Attribution License, which permits unrestricted use, distribution, and reproduction in any medium, provided the original work is properly cited.

The hypothalamus is a source of neural progenitor cells which give rise to different populations of specialized and differentiated cells during brain development. Newly formed neurons in the hypothalamus can synthesize and release various neuropeptides. Although term neuropeptide recently undergoes redefinition, small-size hypothalamic neuropeptides remain major signaling molecules mediating short- and long-term effects on brain development. They represent important factors in neurite growth and formation of neural circuits. There is evidence suggesting that the newly generated hypothalamic neurons may be involved in regulation of metabolism, energy balance, body weight, and social behavior as well. Here we review recent data on the role of hypothalamic neuropeptides in adult neurogenesis and neuritogenesis with special emphasis on the development of food intake and social behavior related brain circuits.

\section{Introduction}

During early developmental periods, rapid proliferation, differentiation, and migration of new progenitor cells occur especially in the hippocampus, subventricular zone, and olfactory bulb [1]. Recent studies suggest that newborn neural cells may be found in the hypothalamus and they produce various neuropeptides [2]. Current evidence indicates that continuous neurogenesis takes place during development of neural system and processes of generation and maturation of neurons extend to adulthood [3]. Many factors influence adult brain neurogenesis such as hormones, growth factors, and neurotransmitters [4]. Newly generated neurons form initial neurites which differentiate into long-distance projections called axons or into multiple short length dendrites. Brain connectivity and promotion of neurite outgrowth are tightly regulated by cytoskeletal components, microtubule proteins, actin-binding proteins, Rho pathway signaling proteins, synaptic scaffolding proteins, adhesion molecules, and locally secreted neuropeptide hormones $[5,6]$. The role of neuropeptides in brain development has been extensively studied and thus certain neuropeptides have been already associated with neurogenesis [7]. Proper time course of generation of specific neuron populations and their interconnections are important factors in hypothalamic development. The role of neuropeptides in neurite growth and formation of neural circuits is far less clear. Many studies suggest that developmental abnormalities in specific hypothalamic circuits are associated with obesity, sleep disorders, anxiety, depression, and autism [8,9]. Growth and guidance of neurites from and to the hypothalamus is essential for understanding their pathogeneses. The ability of neuropeptides to modulate neurogenesis and neurite growth is discussed in the present review.

\section{Neuropeptides}

Neuropeptides represent large and diverse group of molecules responsible for communication among cells in the central nervous system (CNS). Although neuropeptides may be located in the periphery and also play a role in control 
of peripheral functions, their major effects are within the CNS, by taking part in the regulation of thermoregulation, food and water intake, circadian rhythms, and sexual and reproductive behavior. A molecule can be considered as a neuropeptide, when it possesses distinct properties: (1) it is a small-size protein molecule, (2) it is produced and secreted by cells of the nervous system, and (3) it plays a specific role in the regulation of neuronal cells [10]. At least in mammals, neuropeptides are encoded by over 70 genes [10]. The size of the molecule is usually between 3 and 100 amino acids (AC), while more than $75 \%$ of known neuropeptides have a molecule of less than 30 AC [11]. Neuropeptide synthesis takes place mostly in a physiologically inactive form as the pre-pro-molecules. The precursor, before being stored or released from the cell, is typically degraded to short chain of AC through endopeptidases in the Golgi apparatus or directly in the secretory vesicles $[12,13]$. Nevertheless, some neuropeptide molecules undergo further posttranslational modifications, necessary to ensure their stability and full biological activity, such as phosphorylation, acetylation, sulphonation, or removal of their terminal part. Metabolic changes necessary to achieve a fully active form of the neuropeptide are sometimes so intensive that the result leads to an extreme shortening of the peptide chain. Particularly, thyrotropin releasing hormone (TRH) consists only of three amino acids, compared to its much larger pre-pro-form [14]. Neuropeptides are secreted from large dense core vesicles by regulated secretion. They may be stored in vesicles together with other low molecular weight neuropeptides or even with other neurotransmitters [15]. Their secretion is not necessarily limited to the synaptic cleft; however it usually occurs in the close vicinity $[14,16,17]$. There are also reported cases of secretion from the cell body or from dendritic spines [18]. Neuropeptides play a crucial role in cell-to-cell communication by affecting gene expression [19], synaptogenesis [20], and modulation of membrane excitability [21]. Some neuropeptides even may act as neurotransmitters [22]. Despite the often generalized physiological effects of many neuropeptides, time of their biological activity in the circulation is significantly limited. For instance, oxytocin has a half-life in blood of approximately 120 seconds, compared to the half-life in the CNS extracellular space, which is about 20 minutes [23]. Diffusion through the extracellular space and binding to membrane receptors are in a case of robust neuropeptide much slower, however, from the physical-chemical point of view, more solid [24]. Slower modulatory effect on the potential of the postsynaptic membrane is linked to the mechanism of the neuropeptide receptor pathway. Most neuropeptides have their own specific receptor coupled with G-protein. Although the size of neuropeptide molecules is relatively large compared to classical neurotransmitters, affinity to the specific receptors is approximately 1000 -fold higher than that of the neurotransmitter, thus being capable of eliciting a biological response at lower concentrations [21].

\section{Classification of Neuropeptides}

Up to date, the different databases (NeuroPep, NeuroPedia, http://www.neuropeptides.nl/) cover over 5900 neuropeptides divided into large groups $[10,11,25]$. While the number of neuropeptides in vertebrates reaches nearly 2500 [11], it can be expected that the list is still not complete. The division into families may be based on similarities in the gene structure (e.g., calcitonin gene family, F- and Y-amide gene family), molecule structure (e.g., oxytocin/vasopressin family, insulin/insulin-like growth factor (IGF) family), function (e.g., opioid neuropeptide family, adipose neuropeptide family), or localization of neurons producing each neuropeptide (hypothalamic neuropeptide family, hypophyseal neuropeptide family). Many novel neuropeptides remain unclassified. One given peptide is often localized to different brain areas and it is involved in more than one biological function. Neuropeptides expressed in hypothalamic neurons form a large group of well-described peptides with a variety of peripheral (endocrine) and central functions.

\section{Structure of the Hypothalamus}

4.1. Hypothalamic Neuronal Populations. The hypothalamus is an ancient and conserved forebrain area, traditionally divided to lateral, medial, and periventricular part and furthermore to the distinct functional nuclei [26]. Hypothalamic nuclei contain diverse cell populations [27], which can be defined by specific patterns of gene expression, such as ion channels, transcription factors, and neuropeptides (Figure 1). Populations of neurons secreting various neuropeptides located in the lateral hypothalamus play a major role in food intake. In the arcuate nucleus, neurons express orexigenic agouti-related peptide (AgRP), Neuropeptide Y (NPY), and anorexigenic peptides proopiomelanocortin (POMC). Another group of neurons produce peptides promoting food intake-orexin and melanin-concentrating hormone $(\mathrm{MCH})$ [28]. Nevertheless, arcuate hypothalamic neurons that produce proopiomelanocortin (POMC) secrete an anorexic neuropeptide melanocyte-stimulating hormone ( $\alpha$-MSH), a proteolytic product of POMC. Another endogenous peptide product of the POMC represents adrenocorticotropic hormone (ACTH), $\beta$ - and $\gamma$-melanocyte-stimulating hormones ( $\beta$ - and $\gamma-\mathrm{MSH})$, and $\beta$ - endorphin. Located lateral to the arcuate nucleus, the ventromedial nucleus is the major constituent of the mediobasal hypothalamus. Ventromedial nucleus is important in the regulation of sexual behavior and analgesia [29]. Large amount of neuropeptides, such as Substance P, enkephalins, and NPY, is synthesized in the ventromedial nucleus [29]. The periventricular part of the hypothalamus is responsible for secretion of NPY, TRH, somatostatin, leptin, gastrin, and gonadotropin-releasing hormone. Paraventricular and supraoptic nuclei of the hypothalamus contain neurons producing corticotrophinreleasing hormone $(\mathrm{CRH}), \mathrm{TRH}$, oxytocin, and vasopressin (Figure 1).

4.2. Hypothalamic Neuronal Connections. The hypothalamus sends information directly to other brain areas and 


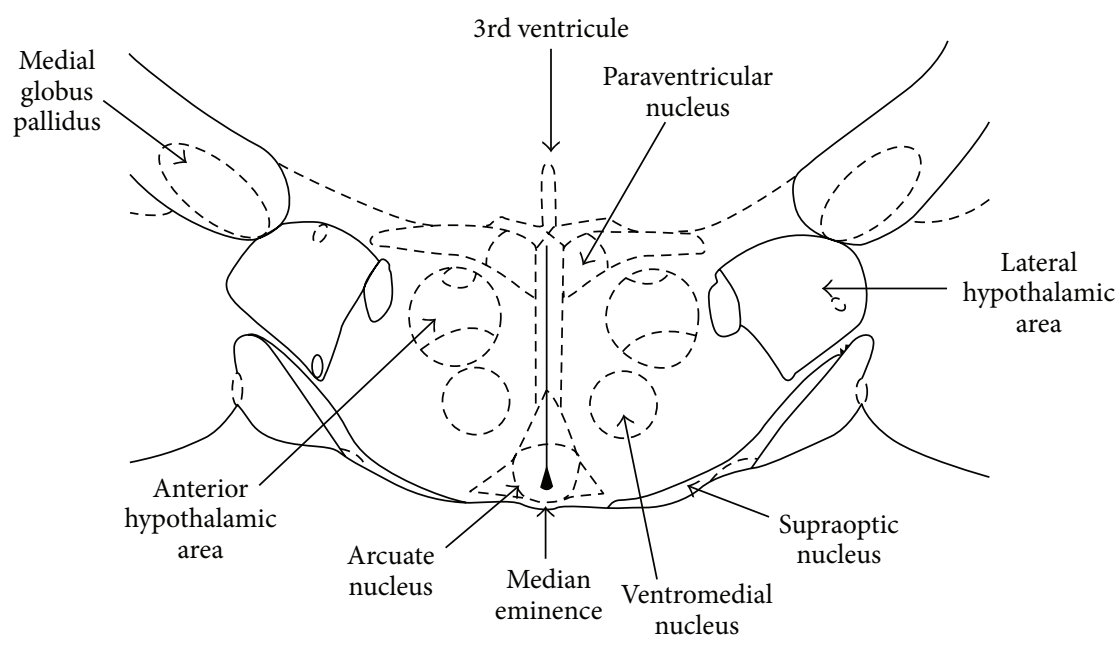

FIGURE 1: Overview of the major hypothalamic nuclei producing neuropeptides. Paraventricular and supraoptic nuclei contain neurons producing corticotrophin-releasing hormone, urocortins, thyrotropin releasing hormone, oxytocin, and vasopressin. Ventromedial nucleus neurons produce Substance P, enkephalins, and Neuropeptide Y. Arcuate nucleus neurons express agouti-related peptide, Neuropeptide Y, proopiomelanocortin, melanocyte-stimulating hormones, adrenocorticotropic hormone-stimulating hormone, and endorphins (modified according to Paxinos and Watson, 1997 [30]).

to periphery by neural projections and indirectly to the blood stream. Neuroendocrine regulation is mediated mostly via hypothalamic-pituitary-adrenal, hypothalamic-pituitarythyroideal, and hypothalamic-pituitary-gonadal axes. Proper transmission of neural signals from periphery to the hypothalamus is mediated by visceral and somatosensory inputs. Furthermore, control of the autonomic nervous system is assured by direct outputs to the brain stem. It is very well documented that neural projections originating or terminating in the hypothalamus are involved in regulation of food, energy, and heat balance. The hypothalamo-neurohypophyseal system plays a fundamental role in the control of fluid and electrolyte balance forming complex neural network responsible for an integrated response [31]. It is also known that olfactory receptor neurons form circuits with hypothalamic subregions [32]. Next, pathways from retina to the suprachiasmatic nucleus of the hypothalamus are involved in regulation of circadian rhythms and light-dark cycle. Projections from the hypothalamus to the cerebral cortex participate in the control of sexual, reproductive, and social behavior $[26,27,33]$.

\section{Development of the Hypothalamus}

The relevant information on functional organization of intraand interhypothalamic circuits has dramatically increased in the last decades. The hypothalamus has complex connections with other brain regions ranging from retina to cortex. These connections are formed during embryonic development; however they are further rearranged later in life under conditions of nutritional state, stress, or lactation [50-52]. Hypothalamic circuits, connections, and pathways are thus dynamically regulated resulting in marked changes of brain plasticity manifested by enhanced neurogenesis and neuritogenesis. It is known that hypothalamic neurogenic niche (hypothalamic proliferating zone) lining the ventral portion of the third ventricle consists of cells with high proliferative activity even in the adult age $[53,54]$. Precursor cells lining the third ventricle are able to receive diverse molecular signals, for example, neuropeptides, and growth factors present in the cerebrospinal fluid. Mounting evidence suggest that hypothalamic neurogenic capacities can be affected in the adult mammalian brain [55]. In addition to production of neurons, shift from neurogenesis to gliogenesis has been shown in the developing hypothalamus [56]. Traditionally, it was believed that most of the hypothalamus is formed in three neurogenetic stages producing neurons that progressively accumulate; however recent studies suggest that hypothalamic progenitor cells have common origin [56]. Nevertheless, it is known that the hypothalamus develops from the rostral diencephalon and cells from various origins migrate to the hypothalamic region during development. Hypothalamic neuron populations are under the control of many intracellular transcriptional factors. The most known are sonic hedgehog protein (Shh) $[56,57]$ and a group of proteins belonging to wingless family (Wnt), which has been long known to be involved in patterning during development [54]. Shh is considered as a morphogen that regulates the dorsoventral patterning of central nervous system. Recent study has demonstrated that Shh coordinates anteroposterior and dorsoventral patterning in the hypothalamus [58]; moreover it has been reported that chemorepulsive effect of Shh repels hypothalamic axons from the ventricular zone of the hypothalamus and results in their growth in fascicules [59]. Differentiated neurons or glia cells cease to express Shh [60]. Wnt signaling is required for neurogenesis and eventually for anterior patterning, including the region that gives rise to the hypothalamus [54, 61]. Newborn cells have been described in the adult hypothalamus, suggesting constitutive neurogenic and cell proliferation responsive to mitogen action [62]. The development of hypothalamic tissue is under control of other morphogenes, namely, bone morphogenetic proteins and fibroblast growth factors (FGF). 
TABLE 1: Effects of small-size neuropeptides on neurogenesis and neuritogenesis.

\begin{tabular}{lcll}
\hline Name & Size of amino acids & Effect on neurogenesis & Effect on neuritogenesis \\
\hline $\begin{array}{l}\text { Orexins (orexin-A) } \\
\begin{array}{l}\text { Melanin-concentrating } \\
\text { hormone (MCH) }\end{array}\end{array}$ & 33 & $\begin{array}{l}\text { Primary hippocampal cells [34] } \\
\uparrow \text { gyrus dentatus [34, 35] }\end{array}$ & $\uparrow$ primary cortical cells [36] \\
$\begin{array}{l}\text { Melanocyte-stimulating } \\
\text { hormone ( } \alpha \text {-MSH, } \beta \text {-MSH) }\end{array}$ & 13 and 18 & $\uparrow$ gyrus dentatus [38, 39] & $\uparrow$ SH-SY5Y cells [37] \\
$\begin{array}{l}\text { Substance P } \\
\text { Enkephalins } \\
\text { (met-enkephalin) }\end{array}$ & 11 & $\uparrow$ spinal neural stem cells [41] & $\uparrow$ dorsal root ganglia neuron culture [40] \\
$\begin{array}{l}\text { Neuropeptide Y (NPY) } \\
\text { Thyrotropin releasing } \\
\text { hormone (TRH) } \\
\begin{array}{l}\text { Corticotrophin-releasing } \\
\text { hormone (CRH) }\end{array}\end{array}$ & 5 & $\uparrow$ SH-SY5Y cells, Neuro-2A cells [42] & $\uparrow$ Neuro-2A cells [42] \\
$\begin{array}{l}\text { Oxytocin } \\
\text { Vasopressin }\end{array}$ & 36 & $\uparrow$ gyrus dentatus [43] & $\uparrow$ dorsal root ganglia neuron culture [44] \\
\hline
\end{tabular}

Neurites in the hypothalamus are guided to their targets by many attractive and repulsive guidance molecules netrins, slits, semaphorins, and ephrins that have been reviewed in the context of autism elsewhere [5]. Complex molecular interactions, including the action of neuropeptide oxytocin, occur at the origin of the hypothalamic region and generation of hypothalamic cell types during development $[63,64]$.

\section{Role of Hypothalamic Neuropeptides in Neurogenesis}

6.1. Adult Hypothalamic Neurogenesis. The presence of immature mitotic neurons in the hypothalamus has been first reported by Evans et al. [65]. Recent evidence for adult hypothalamic neurogenesis has been expanded, which consequently leads to the broad discussion of details on hypothalamic neurogenic cascades, regulatory mechanisms, and potential functions $[66,67]$. Adult-born neurons were found in the rat, mouse, and sheep hypothalamus [68]; however proliferating neural cells in the human hypothalamus has not yet been reliably evidenced. Hypothalamic neurogenic niche has been identified lining the ventral portion of the third ventricle [53]. Moreover, surface of the third ventricle has been suggested as a source of neurogenesis in the adult age and one study has shown that voluntary exercise correlates with proliferation of subependymal cells $[2,69]$. It has been found that median eminence tanycytes (glial cells) generate newborn neurons. Tanycytes represent multipotential cells retaining the morphological features of embryonic glial cells and neural progenitor cells into adulthood [69]. Nevertheless, identity of the hypothalamic neural progenitor cells still remains controversial. It appears that they represent selfrenewing cells that give rise to new tanycytes, astrocytes, and neurons [70]. Immature migrating neurons are highly present in the vicinity of the hypothalamic neurogenic niche [71]. Migrating neurons in the hypothalamus can integrate into functional circuits and modulate brain plasticity. Newly formed neurons in the hypothalamus can synthesize and release various neuropeptides [2]. There is evidence suggesting that the newly generated hypothalamic neurons may be involved in metabolism, energy balance, and body weight $[72,73]$.

\subsection{Hypothalamic Neuropeptides Controlling Neurogenesis.} The potential of certain neuropeptides to affect hippocampal neurogenesis has been extensively reviewed elsewhere [7]; however the involvement of neuropeptides in hypothalamic neurogenesis is less clear. The generation of new cells in the brain has been proved under influence of certain neuropeptides (Table 1). Neuropeptide oxytocin has been reported to stimulate neurogenesis; however its effect was predominately described in the adult hippocampus $[47,74]$. Moreover, oxytocin may affect expression of neurotrophic factors such as brain-derived neurotrophic factor (BDNF) and nerve growth factor (NGF), which represent important regulators of neuronal function [75]. Infusion of BDNF into the lateral ventricle results in generation of new neurons in the hypothalamus of the adult rat [76]. BDNF and many other growth factors and/or neurotrophic factors such as FGF2, ciliary neurotrophic factor (CNTF), vascular endothelial growth factor (VEGF), and transforming growth factor $\alpha$ (TGF- $\alpha$ ) have been shown to regulate neural stem cells and neural progenitor proliferation in the adult rodent brain [77, 78].

One study has reported that neurogenesis occurs in the adult hypothalamus, including areas containing oxytocin and vasopressin producing neurons [74]. Other authors have reported that postnatal neurogenesis occurs in the magnocellular neurons of supraoptic and paraventricular nucleus [79]. They have speculated that different time periods of formation exist for neurons that have a specific function. Moreover, it has been reported that production rate of new neurons expressing vasopressin was positively correlated with postnatal growth of the same hypothalamic region [80]. In agreement with this finding, important role of vasopressin and $\mathrm{CRH}$ in the regulation of hippocampal neurogenesis has 
been suggested [49]. Stem-like cells have been isolated from hypothalamus with the ability to generate neurons and glia producing and secreting neuropeptides including oxytocin [81].

Few studies suggest an association between eating behavior and hypothalamic neurogenesis [28]. This makes a great potential for neuropeptides involved in neurogenesis as neural progenitor cells isolated from fetal rat hypothalamus express NPY, AgRP, and POMC [82]. Peptide melanocortin, one of the POMC products, exhibits control of feeding and energy expenditure, neuroprotection, and neurogenesis through melanocortin-4 receptor subtype (MC4R) [39]. Moreover, melanocortin-induced neurogenesis triggering the Wnt and Shh signaling pathways has been demonstrated in the model of cerebral ischemia [38]. Control of food intake regulated by orexin may include effects on neurogenesis as well. Few studies have suggested that orexin-A is involved in hippocampal neurogenesis [34, 35]. Another neuropeptide, NPY, regulates the biological dynamics of neurogenic niche $[83,84]$ and plays a role in the modulation of adult neurogenesis [7, 85-87]. NPY directly targets certain neural stem cell subtypes (nestin- and doublecortin-positive cells), including proliferation, differentiation, migration, and functional integration of newborn neurons. Moreover, microglia and astrocytes also appear to be responsive to the peptide [43]. NPY directly interacts with another feeding-regulatory peptide ghrelin [88]. Another study has been performed by Chang et al. These authors found that prenatal nicotine exposure stimulates neurogenesis of orexigenic peptide-expressing neurons in the offspring hypothalamus [89]. Systemic ghrelin treatment stimulated neurogenesis in the adult hippocampus in mice [90]. In the hypothalamic neuronal cells, ghrelin may act as a survival factor that preserves mitochondrial integrity and inhibits apoptotic pathways during oxygen-glucose deprivation [91]. Thus, taken together oxytocin, vasopressin, NPY, and ghrelin belong to neuropeptides likely to participate in the regulation of hypothalamic neurogenesis and differentiation. Recently, it has been demonstrated that Substance $P$ increased proliferation of neural stem/progenitor cells in the spinal cord [41]. Although no direct effect of TRH on neurogenesis is so far known, a lot of knowledge has been gained on neurogenic effects of thyroid hormones [92]. Recent study has evidenced that CRH regulates neurogenesis. The same authors have demonstrated that $\mathrm{CRH}$ induced proliferation and protection from apoptosis in the human neuroblastoma cells [46].

\section{Role of Hypothalamic Neuropeptides in Neuritogenesis}

7.1. Hypothalamic Neuritogenesis. Reviews dealing with methodological approaches related to the analysis of hypothalamic circuitry and extensive data on the development of the major axonal tracts coursing through the hypothalamus have been recently published [27, 56, 93]. Neurite outgrowth has been studied in cultures of dissociated hypothalamic cells as well [94, 95]. Sex differences in neuritogenesis in neuronal hypothalamic cultures have been also suggested [96]. The formation of projection pathways in and out of the hypothalamus is critical for a variety of neuroendocrine functions and its postnatal regulation is under control of neuropeptides (Table 1).

\subsection{Hypothalamic Neuropeptides Controlling Food Related Cir-} cuits. Development of certain hypothalamic circuits depends on daily energy and food requirements. Moreover, agedependent formation of intrahypothalamic axonal connection related to regulation of food intake has been extensively described [97]. Recent studies suggest that neuropeptide oxytocin is involved in energy balance control. It has been shown that hypothalamic oxytocin pathways to the brain stem contribute to the reduction of food intake [98, 99]. Oxytocin-producing cells appear early in the development of the hypothalamus [100] and their maturation, and, in particular, their ability to produce oxytocin may influence the formation of hypothalamic circuits and growth of neurites. Several studies suggest that hypothalamic neurons expressing orexigenic and anorexigenic peptides play a role in regulation of neurite growth in early developmental stage [9]. Neurons that express $\alpha$-MSH are particularly important for regulation of hypothalamic development. It has been shown that $\alpha$ MSH promotes neurite elongation through MC4R G-protein coupled receptor [40]. Moreover POMC neurons together with $\alpha$-MSH producing neurons send axonal projections to the brain stem suggesting a functional role in the control of food intake [101]. Melanocortin $\alpha$-MSH has been found to influence the differentiation of neural processes in brain neurons via increase in the levels of neurofilament proteins [102]. Reduction of food intake and body weight regulated by $\alpha-\mathrm{MSH}$ represents a control mechanism for maintenance of energy balance. As neuropeptides represent large group of signaling molecules, they may act on the large number of receptors and share the mechanism of action on neurite extension with other neuropeptides. It has been shown that NPY promotes axonal growth and affects growth cone turning [44]. Another orexigenic peptide orexin-A has been shown to inhibit neurite retraction [36]. The same authors also observed the effect of orexin on neuronal cytoskeleton morphologic changes of actin and vimentin [103]. It has been found that neuropeptide galanin stimulates neurite outgrowth $[44,104]$. Within the hypothalamus, neurons of the suprachiasmatic nucleus contain galanin and galanin mRNA distribution has been described in the arcuate and dorsomedial hypothalamic nuclei as well [105]. Axon tip accumulation of Substance P, NPY, and galanin has been observed in the model of nerve injury suggesting their role in neurite sprouting [106]. Moreover it has been found that ghrelin acts directly on hypothalamic neurons to block axon growth and reduce the overall length of axon extensions [107]. Although not directly related to the topic of the present review, it should be mentioned that recent study demonstrated that gastric peptide ghrelin mediates neural fiber growth in the arcuate nucleus of the hypothalamus during the neonatal period [107]. Development of appetite-related hypothalamic neural projections thus remains complex involving various neuropeptides originating in the central nervous system and periphery as well. 
7.3. Hypothalamic Neuropeptides Controlling Social Behavior Related Circuits. On the basis of functional and anatomical data, comparative studies have described "social behavior network" in mammals that represents the complex neural machinery for the regulation of social behavior [108]. As components, medial amygdala, bed nucleus of the stria terminalis, lateral septum, and ventromedial and anterior hypothalamus have been included to the circuit. These areas are all reciprocally connected and express various neuropeptides and sex steroid hormone receptors as well. Many studies have examined the role of hypothalamic neuropeptides (Figure 1) in social behavior. Recent studies have shown that olfactory receptor neurons participate in polysynaptic circuits with hypothalamic subregions, involving neuropeptides urocortins in the processing of social cues $[32,109]$. It is known for a long time that vasopressin and oxytocin enhance social recognition [110]. Neural mechanisms regulating social cognition and affiliative behavior always include oxytocin action [111]. Traditionally, it is believed that oxytocin and vasopressin are released within the hypothalamic and limbic areas from axons, dendrites, and cell bodies resulting in regulation of mating, reproductive, and affiliative behavior [112]. Particularly detailed review on central oxytocin pathways in the development has been recently published [113]. Embryonic hypothalamus produces immature oxytocin and cells start to generate mature (amidated) oxytocin after birth. Authors suggest that oxytocin axons grow from hypothalamus to forebrain regions and to brain stem/spinal cord after weaning [113]. Individual oxytocin neuronal projections can be found in the bed nucleus of the stria terminalis and the lateral hypothalamic area [114]. Although the bodies of oxytocin neurons are mainly restricted to the hypothalamus, oxytocin fibers are spread throughout the entire brain [111]. Oxytocin increases the firing of inhibitory hippocampal neurons [115]. Recent study has reported that oxytocin is involved in the regulation of social behavior through special cortical circuit [116]. A number of studies suggest that oxytocin modulates social perception, social cognition, and social behavior in humans. Recent reviews have been published dealing with the role of oxytocin and vasopressin in social behavior [117, 118]. Neural circuitry for social cognition depends on oxytocin and vasopressin receptor density in specific brain regions [119, 120]. Link between the individual variation in social behavior and neuropeptidergic systems including oxytocin system has been repeatedly suggested [121]. Oxytocin has sex-specific effects and it can contribute to gregariousness in both sexes in different species [122]. It can be suggested that contribution of specific peptide cell groups in the hypothalamus is important for pair bonds.

\section{Conclusions and Perspectives}

Research during recent years has shown that hypothalamic neural organization continuously changes in response to internal and external stimuli and consequently it results in production of new cells and their differentiation. In this context, it is important to understand the role of hypothalamic neuropeptides in neurogenesis and neuritogenesis. Particularly, small-size neuropeptides may play a role in neuronal proliferation and differentiation influencing growth and guidance of neurites and participating in the formation of neural circuits in early development. Neuropeptides may affect neuronal morphology, cell shape, and arborisation of dendrites as well. Hypothalamic neuropeptides may contribute to the programming of neural progenitor cells. Determination of cell fate in the view of neuropeptide production and secretion is important point for development of functional neural circuits. Although many studies mentioned in the present review show and discuss altered number of neurons and enhanced neurogenesis, conclusions should be considered carefully as functional contribution of neuropeptides is always related to concerted effects of different signaling molecules. Moreover, short-term and long-term effects of neuropeptides differ greatly and may have opposite or variable actions on brain tissue. According to the literature, many neuropeptides show trophic effects, often site and time specific. Distribution of neuropeptides and precise knowledge of their transportation from the hypothalamus to the other brain regions are therefore crucial. Further studies should focus on neuropeptide pathways and their changes during development. Conditional formation of neuronal circuits is extremely important. Effects of neuropeptides have fairly complex consequences ranging from modulation of food intake to establishment of social bonds. Recent studies have already shown the role of oxytocin and other hypothalamic neuropeptides in early development. Maintenance of balance in the orexigenic and anorexigenic hypothalamic neuropeptides is especially important in the context of maximized energy intake and mass gain during early stages of development. Nevertheless, understanding mechanisms that facilitate the formation of neural circuits mediating food intake and establishment of social bonds may help to resolve diagnosis and treatment of developmental diseases.

\section{Abbreviations}

\section{AC: $\quad$ Amino acid}

ACTH: Adrenocorticotropic hormone

AgRP: Agouti-related peptide

BDNF: Brain-derived neurotrophic factor

CNS: Central nervous system

CNTF: Ciliary neurotrophic factor

$\mathrm{CRH}$ : Corticotrophin-releasing hormone

FGF: Fibroblast growth factor

IGF: Insulin-like growth factor

$\mathrm{MCH}$ : Melanin-concentrating hormone

MC4R: Melanocortin-4 receptor

MSH: Melanocyte-stimulating hormone

NGF: Nerve growth factor

NPY: Neuropeptide Y

POMC: Proopiomelanocortin

Shh: Sonic hedgehog protein

TGF- $\alpha$ : Transforming growth factor $\alpha$

TRH: Thyrotropin releasing hormone

VEGF: Vascular endothelial growth factor

Wnt: Wingless family proteins. 


\section{Conflict of Interests}

The authors declare that there is no conflict of interests regarding the publication of this paper.

\section{Acknowledgments}

The work was supported by the Slovak Research and Development Agency Projects APVV-0253-10 and APVV-025411 and by the Grant Agency of Ministry of Education and Slovak Academy of Sciences (VEGA) Projects 1/0052/15 and 2/0119/15

\section{References}

[1] A. Alvarez-Buylla and J. M. García-Verdugo, "Neurogenesis in adult subventricular zone," The Journal of Neuroscience, vol. 22, no. 3, pp. 629-634, 2002.

[2] E. Rojczyk-Gołębiewska, A. Pałasz, and R. Wiaderkiewicz, "Hypothalamic subependymal niche: a novel site of the adult neurogenesis," Cellular and Molecular Neurobiology, vol. 34, no. 5, pp. 631-642, 2014.

[3] Y. Gu, S. Janoschka, and S. Ge, "Neurogenesis and hippocampal plasticity in adult brain," Current Topics in Behavioral Neurosciences, vol. 15, pp. 31-48, 2013.

[4] G. A. Elder, R. De Gasperi, and M. A. Gama Sosa, "Research update: neurogenesis in adult brain and neuropsychiatric disorders," Mount Sinai Journal of Medicine, vol. 73, no. 7, pp. 931940, 2006.

[5] J. Bakos, Z. Bacova, S. G. Grant, A. M. Castejon, and D. Ostatnikova, "Are molecules involved in neuritogenesis and axon guidance related to autism pathogenesis?" NeuroMolecular Medicine, vol. 17, no. 3, pp. 297-304, 2015.

[6] F. Polleux and W. Snider, "Initiating and growing an axon," Cold Spring Harbor Perspectives in Biology, vol. 2, no. 4, Article ID a001925, 2010.

[7] M. J. Zaben and W. P. Gray, "Neuropeptides and hippocampal neurogenesis," Neuropeptides, vol. 47, no. 6, pp. 431-438, 2013.

[8] J. Biran, M. Tahor, E. Wircer, and G. Levkowitz, "Role of developmental factors in hypothalamic function," Frontiers in Neuroanatomy, vol. 9, article 27, 2015.

[9] H. MacKay and A. Abizaid, "Embryonic development of the hypothalamic feeding circuitry: transcriptional, nutritional, and hormonal influences," Molecular Metabolism, vol. 3, no. 9, pp. 813-822, 2014.

[10] J. P. Burbach, "What are neuropeptides?" Methods in Molecular Biology, vol. 789, pp. 1-36, 2011.

[11] Y. Wang, M. Wang, S. Yin et al., "NeuroPep: a comprehensive resource of neuropeptides," Database, vol. 2015, Article ID bav038, 2015.

[12] S. J. Husson, T. Janssen, G. Baggerman et al., "Impaired processing of FLP and NLP peptides in carboxypeptidase E (EGL-21)-deficient Caenorhabditis elegans as analyzed by mass spectrometry," Journal of Neurochemistry, vol. 102, no. 1, pp. 246-260, 2007.

[13] C. Kienzle and J. von Blume, "Secretory cargo sorting at the trans-Golgi network," Trends in Cell Biology, vol. 24, no. 10, pp. 584-593, 2014.

[14] C. Salio, L. Lossi, F. Ferrini, and A. Merighi, "Neuropeptides as synaptic transmitters," Cell and Tissue Research, vol. 326, no. 2, pp. 583-598, 2006.
[15] A. Merighi, "Costorage and coexistence of neuropeptides in the mammalian CNS," Progress in Neurobiology, vol. 66, no. 3, pp. 161-190, 2002.

[16] T. Karhunen, F. S. Vilim, V. Alexeeva, K. R. Weiss, and P. J. Church, "Targeting of peptidergic vesicles in cotransmitting terminals," The Journal of Neuroscience, vol. 21, no. 3, p. RC127, 2001.

[17] M. P. Nusbaum, "Regulating peptidergic modulation of rhythmically active neural circuits," Brain, Behavior and Evolution, vol. 60, no. 6, pp. 378-387, 2002.

[18] M. Ludwig and G. Leng, "Dendritic peptide release and peptidedependent behaviours," Nature Reviews Neuroscience, vol. 7, no. 2, pp. 126-136, 2006.

[19] R. Landgraf and I. D. Neumann, "Vasopressin and oxytocin release within the brain: a dynamic concept of multiple and variable modes of neuropeptide communication," Frontiers in Neuroendocrinology, vol. 25, no. 3-4, pp. 150-176, 2004.

[20] D. T. Theodosis, D. B. Chapman, C. Montagnese, D. A. Poulain, and J. F. Morris, "Structural plasticity in the hypothalamic supraoptic nucleus at lactation affects oxytocin-, but not vasopressin-secreting neurones," Neuroscience, vol. 17, no. 3, pp. 661-678, 1986.

[21] A. Merighi, C. Salio, F. Ferrini, and L. Lossi, "Neuromodulatory function of neuropeptides in the normal CNS," Journal of Chemical Neuroanatomy, vol. 42, no. 4, pp. 276-287, 2011.

[22] T. Hökfelt, T. Bartfai, and F. Bloom, "Neuropeptides: opportunities for drug discovery," The Lancet Neurology, vol. 2, no. 8, pp. 463-472, 2003.

[23] W. B. J. Mens, A. Witter, and T. B. Van Wimersma Greidanus, "Penetration of neurohypophyseal hormones from plasma into cerebrospinal fluid (CSF): half-times of disappearance of these neuropeptides from CSF," Brain Research, vol. 262, no. 1, pp. 143149, 1983.

[24] W. De Haes, E. Van Sinay, G. Detienne, L. Temmerman, L. Schoofs, and K. Boonen, "Functional neuropeptidomics in invertebrates," Biochimica et Biophysica Acta-Proteins and Proteomics, vol. 1854, no. 7, pp. 812-826, 2014.

[25] Y. Kim, S. Bark, V. Hook, and N. Bandeira, "NeuroPedia: neuropeptide database and spectral library," Bioinformatics, vol. 27, no. 19, pp. 2772-2773, 2011.

[26] P. Y. Risold, R. H. Thompson, and L. W. Swanson, "The structural organization of connections between hypothalamus and cerebral cortex," Brain Research Reviews, vol. 24, no. 2-3, pp. 197-254, 1997.

[27] S. M. Sternson, "Hypothalamic survival circuits: blueprints for purposive behaviors," Neuron, vol. 77, no. 5, pp. 810-824, 2013.

[28] L. Sousa-Ferreira, L. P. de Almeida, and C. Cavadas, "Role of hypothalamic neurogenesis in feeding regulation," Trends in Endocrinology and Metabolism, vol. 25, no. 2, pp. 80-88, 2014.

[29] K. M. McClellan, K. L. Parker, and S. Tobet, "Development of the ventromedial nucleus of the hypothalamus," Frontiers in Neuroendocrinology, vol. 27, no. 2, pp. 193-209, 2006.

[30] G. Paxinos and C. Watson, The Rat Brain in Stereotaxic Coordinates, Academic Press, San Diego, Calif, USA, 1997.

[31] J. Antunes-Rodrigues, M. de Castro, L. L. K. Elias, M. M. Valença, and S. M. McCann, "Neuroendocrine control of body fluid metabolism," Physiological Reviews, vol. 84, no. 1, pp. 169208, 2004.

[32] U. Boehm, Z. Zou, and L. B. Buck, "Feedback loops link odor and pheromone signaling with reproduction," Cell, vol. 123, no. 4, pp. 683-695, 2005. 
[33] L. W. Swanson, "Cerebral hemisphere regulation of motivated behavior," Brain Research, vol. 886, no. 1-2, pp. 113-164, 2000.

[34] N. Ito, T. Yabe, Y. Gamo et al., "I.c.v. administration of orexin-A induces an antidepressive-like effect through hippocampal cell proliferation," Neuroscience, vol. 157, no. 4, pp. 720-732, 2008.

[35] X. Zhao, R. X. Zhang, S. Tang et al., "Orexin-A-induced ERK1/2 activation reverses impaired spatial learning and memory in pentylenetetrazol-kindled rats via OX1R-mediated hippocampal neurogenesis," Peptides, vol. 54, pp. 140-147, 2014.

[36] K. Björnström, D. Turina, T. Strid, T. Sundqvist, and C. Eintrei, "Orexin A inhibits propofol-induced neurite retraction by a phospholipase $\mathrm{D} /$ protein kinase $\mathrm{C} \varepsilon$-dependent mechanism in neurons," PLoS ONE, vol. 9, no. 5, Article ID e97129, 2014.

[37] N. Cotta-Grand, C. Rovère, A. Guyon, A. Cervantes, F. Brau, and J.-L. Nahon, "Melanin-concentrating hormone induces neurite outgrowth in human neuroblastoma SH-SY5Y cells through p53 and MAPKinase signaling pathways," Peptides, vol. 30, no. 11, pp. 2014-2024, 2009.

[38] L. Spaccapelo, M. Galantucci, L. Neri et al., "Up-regulation of the canonical Wnt-3A and Sonic hedgehog signaling underlies melanocortin-induced neurogenesis after cerebral ischemia," European Journal of Pharmacology, vol. 707, no. 1-3, pp. 78-86, 2013.

[39] D. Giuliani, D. Zaffe, A. Ottani et al., "Treatment of cerebral ischemia with melanocortins acting at MC4 receptors induces marked neurogenesis and long-lasting functional recovery," Acta Neuropathologica, vol. 122, no. 4, pp. 443-453, 2011.

[40] K. Tanabe, K. Gamo, S. Aoki, K. Wada, and H. Kiyama, "Melanocortin receptor 4 is induced in nerve-injured motor and sensory neurons of mouse," Journal of Neurochemistry, vol. 101, no. 4, pp. 1145-1152, 2007.

[41] K.-T. Kim, H.-J. Kim, D.-C. Cho, J.-S. Bae, and S.-W. Park, "Substance P stimulates proliferation of spinal neural stem cells in spinal cord injury via the mitogen-activated protein kinase signaling pathway," The Spine Journal, vol. 15, no. 9, pp. 20552065, 2015.

[42] E.-M. Georganta, L. Tsoutsi, M. Gaitanou, and Z. Georgoussi, " $\delta$-Opioid receptor activation leads to neurite outgrowth and neuronal differentiation via a STAT5B-G $\alpha$ i/o pathway," Journal of Neurochemistry, vol. 127, no. 3, pp. 329-341, 2013.

[43] M. C. Geloso, V. Corvino, V. Di Maria, E. Marchese, and F. Michetti, "Cellular targets for neuropeptide Y-mediated control of adult neurogenesis," Frontiers in Cellular Neuroscience, vol. 9, article 85, 2015.

[44] S. D. Sanford, J. C. Gatlin, T. Hökfelt, and K. H. Pfenninger, "Growth cone responses to growth and chemotropic factors," European Journal of Neuroscience, vol. 28, no. 2, pp. 268-278, 2008.

[45] Y. Iwasaki, K. Ikeda, T. Shiojima, and M. Kinoshita, “TRH analogue, TA-0910 (3-methyl-(s)-5,6-dihydroorotyl-L-histidylL-prolinamide) enhances neurite outgrowth in rat embryo ventral spinal cord in vitro," Journal of the Neurological Sciences, vol. 112, no. 1-2, pp. 147-151, 1992.

[46] Y. Koutmani, P. K. Politis, M. Elkouris et al., "Corticotropinreleasing hormone exerts direct effects on neuronal progenitor cells: implications for neuroprotection," Molecular Psychiatry, vol. 18, no. 3, pp. 300-307, 2013.

[47] B. Leuner, J. M. Caponiti, and E. Gould, "Oxytocin stimulates adult neurogenesis even under conditions of stress and elevated glucocorticoids," Hippocampus, vol. 22, no. 4, pp. 861-868, 2012.

[48] Z. Lestanova, Z. Bacova, A. Kiss, T. Havranek, V. Strbak, and J. Bakos, "Oxytocin increases neurite length and expression of cytoskeletal proteins associated with neuronal growth," Journal of Molecular Neuroscience, 2015.

[49] R. Alonso, G. Griebel, G. Pavone, J. Stemmelin, G. Le Fur, and P. Soubrié, "Blockade of $\mathrm{CRF}_{1}$ or $\mathrm{V}_{1 b}$ receptors reverses stress-induced suppression of neurogenesis in a mouse model of depression," Molecular Psychiatry, vol. 9, no. 3, pp. 278-286, 2004.

[50] S. Pinto, A. G. Roseberry, H. Liu et al., "Rapid rewiring of arcuate nucleus feeding circuits by leptin," Science, vol. 304, no. 5667, pp. 110-115, 2004.

[51] S. M. Sternson, G. M. G. Shepherd, and J. M. Friedman, “Topographic mapping of $\mathrm{VMH} \rightarrow$ arcuate nucleus microcircuits and their reorganization by fasting," Nature Neuroscience, vol. 8, no. 10, pp. 1356-1363, 2005.

[52] D. T. Theodosis, R. Piet, D. A. Poulain, and S. H. R. Oliet, "Neuronal, glial and synaptic remodeling in the adult hypothalamus: functional consequences and role of cell surface and extracellular matrix adhesion molecules," Neurochemistry International, vol. 45, no. 4, pp. 491-501, 2004.

[53] D. A. Lee, J. L. Bedont, T. Pak et al., "Tanycytes of the hypothalamic median eminence form a diet-responsive neurogenic niche," Nature Neuroscience, vol. 15, no. 5, pp. 700-702, 2012.

[54] J. L. Bedont, E. A. Newman, and S. Blackshaw, "Patterning, specification, and differentiation in the developing hypothalamus," Wiley Interdisciplinary Reviews: Developmental Biology, vol. 4, no. 5, pp. 445-468, 2015.

[55] M. Migaud, L. Butrille, and M. Batailler, "Seasonal regulation of structural plasticity and neurogenesis in the adult mammalian brain: focus on the sheep hypothalamus," Frontiers in Neuroendocrinology, vol. 37, pp. 146-157, 2015.

[56] G. Alvarez-Bolado, F. A. Paul, and S. Blaess, "Sonic hedgehog lineage in the mouse hypothalamus: from progenitor domains to hypothalamic regions," Neural Development, vol. 7, article 4, 2012.

[57] S. Croizier, S. Chometton, D. Fellmann, and P.-Y. Risold, "Characterization of a mammalian prosencephalic functional plan," Frontiers in Neuroanatomy, vol. 8, article 161, 2015.

[58] N.-E. Szabó, T. Zhao, M. Çankaya, T. Theil, X. Zhou, and G. Alvarez-Bolado, "Role of neuroepithelial Sonic hedgehog in hypothalamic patterning," The Journal of Neuroscience, vol. 29, no. 21, pp. 6989-7002, 2009.

[59] F. Liu, M. Placzek, and H. Xu, "Axon guidance effect of classical morphogens Shh and BMP7 in the hypothalamo-pituitary system," Neuroscience Letters, vol. 553, pp. 104-109, 2013.

[60] S. Blaess, N. Szabö, R. Haddad-Tövolli, X. Zhou, and G. ÁlvarezBolado, "Sonic hedgehog signaling in the development of the mouse hypothalamus," Frontiers in Neuroanatomy, vol. 8, article 156, 2014.

[61] J. E. Lee, S.-F. Wu, L. M. Goering, and R. I. Dorsky, "Canonical Wnt signaling through Lef1 is required for hypothalamic neurogenesis," Development, vol. 133, no. 22, pp. 4451-4461, 2006.

[62] M. V. Kokoeva, H. Yin, and J. S. Flier, "Evidence for constitutive neural cell proliferation in the adult murine hypothalamus," Journal of Comparative Neurology, vol. 505, no. 2, pp. 209-220, 2007.

[63] T. Matsuzaki, T. Iwasa, M. Munkhzaya et al., "Developmental changes in hypothalamic oxytocin and oxytocin receptor mRNA expression and their sensitivity to fasting in male and female rats," International Journal of Developmental Neuroscience, vol. 41, pp. 105-109, 2015. 
[64] F. T. Merkle, A. Maroof, T. Wataya et al., "Generation of neuropeptidergic hypothalamic neurons from human pluripotent stem cells," Development, vol. 142, no. 4, pp. 633-643, 2015.

[65] J. Evans, C. Sumners, J. Moore et al., "Characterization of mitotic neurons derived from adult rat hypothalamus and brain stem," Journal of Neurophysiology, vol. 87, no. 2, pp. 1076-1085, 2002.

[66] T.-F. Yuan and O. Arias-Carrión, "Adult neurogenesis in the hypothalamus: evidence, functions and implications," CNS and Neurological Disorders-Drug Targets, vol. 10, no. 4, pp. 433439, 2011.

[67] R. Maggi, J. Zasso, and L. Conti, "Neurodevelopmental origin and adult neurogenesis of the neuroendocrine hypothalamus," Frontiers in Cellular Neuroscience, vol. 8, article 440, 2015.

[68] M. Batailler, L. Derouet, L. Butruille, and M. Migaud, "Sensitivity to the photoperiod and potential migratory features of neuroblasts in the adult sheep hypothalamus," Brain Structure and Function, pp. 1-5, 2015.

[69] A. Niwa, M. Nishibori, S. Hamasaki et al., "Voluntary exercise induces neurogenesis in the hypothalamus and ependymal lining of the third ventricle," Brain Structure and Function, pp. 1-14, 2015.

[70] S. C. Robins, I. Stewart, D. E. McNay et al., " $\alpha$-Tanycytes of the adult hypothalamic third ventricle include distinct populations of FGF-responsive neural progenitors," Nature Communications, vol. 4, article 2049, 2013.

[71] M. Batailler, M. Droguerre, M. Baroncini, C. Fontaine, V. Prevot, and M. Migaud, "DCX-expressing cells in the vicinity of the hypothalamic neurogenic niche: a comparative study between mouse, sheep, and human tissues," Journal of Comparative Neurology, vol. 522, no. 8, pp. 1966-1985, 2014.

[72] M. Bolborea and N. Dale, "Hypothalamic tanycytes: potential roles in the control of feeding and energy balance," Trends in Neurosciences, vol. 36, no. 2, pp. 91-100, 2013.

[73] D. A. Lee, S. Yoo, T. Pak et al., "Dietary and sex-specific factors regulate hypothalamic neurogenesis in young adult mice," Frontiers in Neuroscience, vol. 8, article 157, Article ID Article 157, 2014.

[74] A. D. Raymond, N. N. A. Kucherepa, K. R. S. Fisher, W. G. Halina, and G. D. Partlow, "Neurogenesis of oxytocincontaining neurons in the paraventricular nucleus (PVN) of the female pig in 3 reproductive states: puberty gilts, adult gilts and lactating sows," Brain Research, vol. 1102, no. 1, pp. 44-51, 2006.

[75] T. Havranek, M. Zatkova, Z. Lestanova et al., "Intracerebroventricular oxytocin administration in rats enhances object recognition and increases expression of neurotrophins, microtubuleassociated protein 2, and synapsin I," Journal of Neuroscience Research, vol. 93, no. 6, pp. 893-901, 2015.

[76] V. Pencea, K. D. Bingaman, S. J. Wiegand, and M. B. Luskin, "Infusion of brain-derived neurotrophic factor into the lateral ventricle of the adult rat leads to new neurons in the parenchyma of the striatum, septum, thalamus, and hypothalamus," The Journal of Neuroscience, vol. 21, no. 17, pp. 6706-6717, 2001.

[77] T. Hagg, "Molecular regulation of adult CNS neurogenesis: an integrated view," Trends in Neurosciences, vol. 28, no. 11, pp. 589595, 2005.

[78] P. M. Lledo, M. Alonso, and M. S. Grubb, "Adult neurogenesis and functional plasticity in neuronal circuits," Nature Reviews Neuroscience, vol. 7, no. 3, pp. 179-193, 2006.

[79] J. Iqbal, J. K. Elmquist, L. R. Ross, M. R. Ackermann, and C. D. Jacobson, "Postnatal neurogenesis of the hypothalamic paraventricular and supraoptic nuclei in the brazilian opossum brain," Developmental Brain Research, vol. 85, no. 2, pp. 151-160, 1995.

[80] S. L. Rankin, G. D. Partlow, R. D. McCurdy, E. D. Giles, and K. R. S. Fisher, "Postnatal neurogenesis in the vasopressin and oxytocin-containing nucleus of the pig hypothalamus," Brain Research, vol. 971, no. 2, pp. 189-196, 2003.

[81] E. A. Markakis, T. D. Palmer, L. Randolph-Moore, P. Rakic, and F. H. Gage, "Novel neuronal phenotypes from neural progenitor cells," The Journal of Neuroscience, vol. 24, no. 12, pp. 2886-2897, 2004.

[82] L. Sousa-Ferreira, A. R. Álvaro, C. Aveleira et al., "Proliferative hypothalamic neurospheres express NPY, AGRP, POMC, CART and Orexin-A and differentiate to functional neurons," PLoS ONE, vol. 6, no. 5, Article ID e19745, 2011.

[83] G. Kempermann, L. Wiskott, and F. H. Gage, "Functional significance of adult neurogenesis," Current Opinion in Neurobiology, vol. 14, no. 2, pp. 186-191, 2004.

[84] C. Zhao, W. Deng, and F. H. Gage, "Mechanisms and functional implications of adult neurogenesis," Cell, vol. 132, no. 4, pp. 645660, 2008.

[85] W. P. Gray, "Neuropeptide Y signalling on hippocampal stem cells in health and disease," Molecular and Cellular Endocrinology, vol. 288, no. 1-2, pp. 52-62, 2008.

[86] M. Decressac and R. A. Barker, "Neuropeptide Y and its role in CNS disease and repair," Experimental Neurology, vol. 238, no. 2, pp. 265-272, 2012.

[87] J. O. Malva, S. Xapelli, S. Baptista et al., "Multifaces of neuropeptide $\mathrm{Y}$ in the brain-neuroprotection, neurogenesis and neuroinflammation," Neuropeptides, vol. 46, no. 6, pp. 299-308, 2012.

[88] D. Kohno, H.-Z. Gao, S. Muroya, S. Kikuyama, and T. Yada, "Ghrelin directly interacts with neuropeptide-Y-containing neurons in the rat arcuate nucleus: $\mathrm{Ca}^{2+}$ signaling via protein kinase $\mathrm{A}$ and $\mathrm{N}$-type channel-dependent mechanisms and cross-talk with leptin and orexin," Diabetes, vol. 52, no. 4, pp. 948-956, 2003.

[89] G.-Q. Chang, O. Karatayev, and S. F. Leibowitz, "Prenatal exposure to nicotine stimulates neurogenesis of orexigenic peptide-expressing neurons in hypothalamus and amygdala," The Journal of Neuroscience, vol. 33, no. 34, pp. 13600-13611, 2013.

[90] Z. Zhao, H. Liu, K. Xiao et al., “Ghrelin administration enhances neurogenesis but impairs spatial learning and memory in adult mice," Neuroscience, vol. 257, pp. 175-185, 2014.

[91] H. Chung, E. Kim, D. H. Lee et al., "Ghrelin inhibits apoptosis in hypothalamic neuronal cells during oxygen-glucose deprivation," Endocrinology, vol. 148, no. 1, pp. 148-159, 2007.

[92] R. Kapoor, S. E. Fanibunda, L. A. Desouza, S. K. Guha, and V. A. Vaidya, "Perspectives on thyroid hormone action in adult neurogenesis," Journal of Neurochemistry, vol. 133, no. 5, pp. 599-616, 2015.

[93] G. Alvarez-Bolado, V. Grinevich, and L. Puelles, "Editorial: development of the hypothalamus," Frontiers in Neuroanatomy, vol. 9, article 83, 2015.

[94] S. G. Bouret, S. J. Draper, and R. B. Simerly, "Trophic action of leptin on hypothalamic neurons that regulate feeding," Science, vol. 304, no. 5667, pp. 108-110, 2004.

[95] H. Díaz, A. Lorenzo, H. F. Carrer, and A. Caceres, “Time lapse study of neurite growth in hypothalamic dissociated neurons in culture: sex differences and estrogen effects," Journal of Neuroscience Research, vol. 33, no. 2, pp. 266-281, 1992. 
[96] M. J. Scerbo, A. Freire-Regatillo, C. D. Cisternas et al., "Neurogenin 3 mediates sex chromosome effects on the generation of sex differences in hypothalamic neuronal development," Frontiers in Cellular Neuroscience, vol. 8, article 188, 2014.

[97] S. G. Bouret, S. J. Draper, and R. B. Simerly, "Formation of projection pathways from the arcuate nucleus of the hypothalamus to hypothalamic regions implicated in the neural control of feeding behavior in mice," The Journal of Neuroscience, vol. 24, no. 11, pp. 2797-2805, 2004.

[98] Y. Maejima, R. S. Rita, P. Santoso et al., "Nasal oxytocin administration reduces food intake without affecting locomotor activity and glycemia with c-Fos induction in limited brain areas," Neuroendocrinology, vol. 101, no. 1, pp. 35-44, 2015.

[99] E. T. Uchoa, D. S. Zahm, B. de Carvalho Borges, R. Rorato, J. Antunes-Rodrigues, and L. L. K. Elias, "Oxytocin projections to the nucleus of the solitary tract contribute to the increased meal-related satiety responses in primary adrenal insufficiency," Experimental Physiology, vol. 98, no. 10, pp. 1495-1504, 2013.

[100] E. Madarasz, Z. Kornyei, D. A. Poulain, and D. T. Theodosis, "Development of oxytocinergic neurons in monolayer cultures derived from embryonic, fetal and postnatal rat hypothalami," Journal of Neuroendocrinology, vol. 4, no. 4, pp. 433-439, 1992.

[101] H. Zheng, L. M. Patterson, C. J. Rhodes et al., "A potential role for hypothalamomedullary POMC projections in leptininduced suppression of food intake," American Journal of Physiology-Regulatory Integrative and Comparative Physiology, vol. 298, no. 3, pp. R720-R728, 2010.

[102] V. Kistler-Heer, M. Schlumpf, and W. Lichtensteiger, "Melanocortin and $\mathrm{MCH}$ precursor-derived NEI effects on striatummidbrain co-cultures," Peptides, vol. 19, no. 8, pp. 1317-1327, 1998.

[103] D. Turina, H. Gerhardsson, and K. Bjornstrom, "Orexin a reverses propofoland thiopental induced cytoskeletal rearrangement in rat neurons," Journal of Physiology and Pharmacology, vol. 65, no. 4, pp. 531-541, 2014.

[104] S.-A. Hobson, F. E. Holmes, N. C. H. Kerr, R. J. P. Pope, and D. Wynick, "Mice deficient for galanin receptor 2 have decreased neurite outgrowth from adult sensory neurons and impaired pain-like behavior," Journal of Neurochemistry, vol. 99, no. 3, pp. 1000-1010, 2006.

[105] E. Kuteeva, L. Calza, K. Holmberg, E. Theodorsson, S. O. Ögren, and T. Hökfelt, "Distribution of galanin and galanin transcript in the brain of a galanin-overexpressing transgenic mouse," Journal of Chemical Neuroanatomy, vol. 28, no. 4, pp. 185-216, 2004.

[106] D. W. Zochodne, C. Cheng, M. Miampamba, K. Hargreaves, and K. A. Sharkey, "Peptide accumulations in proximal endbulbs of transected axons," Brain Research, vol. 902, no. 1, pp. 40-50, 2001.

[107] S. M. Steculorum, G. Collden, B. Coupe et al., "Neonatal ghrelin programs development of hypothalamic feeding circuits," The Journal of Clinical Investigation, vol. 125, no. 2, pp. 846-858, 2015.

[108] J. L. Goodson and M. A. Kingsbury, "What's in a name? Considerations of homologies and nomenclature for vertebrate social behavior networks," Hormones and Behavior, vol. 64, no. 1, pp. 103-112, 2013.

[109] J. M. Deussing, J. Breu, C. Kühne et al., "Urocortin 3 modulates social discrimination abilities via corticotropin-releasing hormone receptor type 2," The Journal of Neuroscience, vol. 30, no. 27, pp. 9103-9116, 2010.
[110] E. E. Storm and L. H. Tecott, "Social circuits: peptidergic regulation of mammalian social behavior," Neuron, vol. 47, no. 4, pp. 483-486, 2005.

[111] H. E. Ross and L. J. Young, "Oxytocin and the neural mechanisms regulating social cognition and affiliative behavior," Frontiers in Neuroendocrinology, vol. 30, no. 4, pp. 534-547, 2009.

[112] I. D. Neumann and R. Landgraf, "Balance of brain oxytocin and vasopressin: implications for anxiety, depression, and social behaviors," Trends in Neurosciences, vol. 35, no. 11, pp. 649-659, 2012.

[113] V. Grinevich, M. G. Desarménien, B. Chini, M. Tauber, and F. Muscatelli, "Ontogenesis of oxytocin pathways in the mammalian brain: late maturation and psychosocial disorders," Frontiers in Neuroanatomy, vol. 8, article 164, 2015.

[114] Z. Wang, L. Zhou, T. J. Hulihan, and T. R. Insel, "Immunoreactivity of central vasopressin and oxytocin pathways in microtine rodents: a quantitative comparative study," Journal of Comparative Neurology, vol. 366, no. 4, pp. 726-737, 1996.

[115] S. F. Owen, S. N. Tuncdemir, P. L. Bader, N. N. Tirko, G. Fishell, and R. W. Tsien, "Oxytocin enhances hippocampal spike transmission by modulating fast-spiking interneurons," Nature, vol. 500, no. 7463, pp. 458-462, 2013.

[116] M. Nakajima, A. Görlich, and N. Heintz, "Oxytocin modulates female sociosexual behavior through a specific class of prefrontal cortical interneurons," Cell, vol. 159, no. 2, pp. 295-305, 2014.

[117] M. Heinrichs and G. Domes, "Neuropeptides and social behaviour: effects of oxytocin and vasopressin in humans," Progress in Brain Research, vol. 170, pp. 337-350, 2008.

[118] A. Meyer-Lindenberg, G. Domes, P. Kirsch, and M. Heinrichs, "Oxytocin and vasopressin in the human brain: social neuropeptides for translational medicine," Nature Reviews Neuroscience, vol. 12, no. 9, pp. 524-538, 2011.

[119] S. M. Freeman, K. Inoue, A. L. Smith, M. M. Goodman, and L. J. Young, "The neuroanatomical distribution of oxytocin receptor binding and mRNA in the male rhesus macaque (Macaca mulatta)," Psychoneuroendocrinology, vol. 45, pp. 128-141, 2014.

[120] D. E. Olazábal and L. J. Young, "Species and individual differences in juvenile female alloparental care are associated with oxytocin receptor density in the striatum and the lateral septum," Hormones and Behavior, vol. 49, no. 5, pp. 681-687, 2006.

[121] F. Calcagnoli, S. F. de Boer, D. I. Beiderbeck, M. Althaus, J. M. Koolhaas, and I. D. Neumann, "Local oxytocin expression and oxytocin receptor binding in the male rat brain is associated with aggressiveness," Behavioural Brain Research, vol. 261, pp. 315-322, 2014.

[122] A. M. Kelly and J. L. Goodson, "Hypothalamic oxytocin and vasopressin neurons exert sex-specific effects on pair bonding, gregariousness, and aggression in finches," Proceedings of the National Academy of Sciences of the United States of America, vol. 111, no. 16, pp. 6069-6074, 2014. 

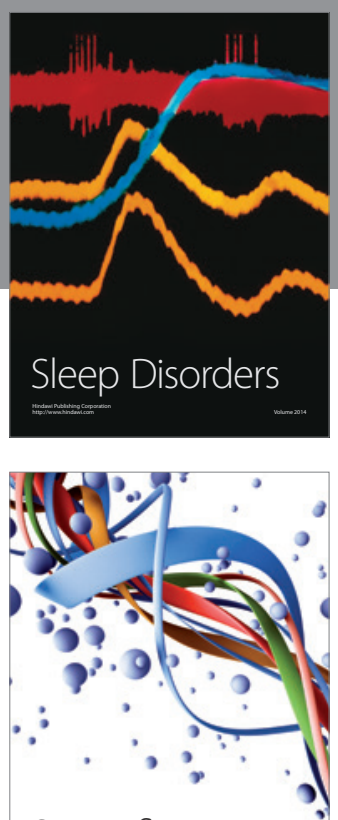

Scientifica
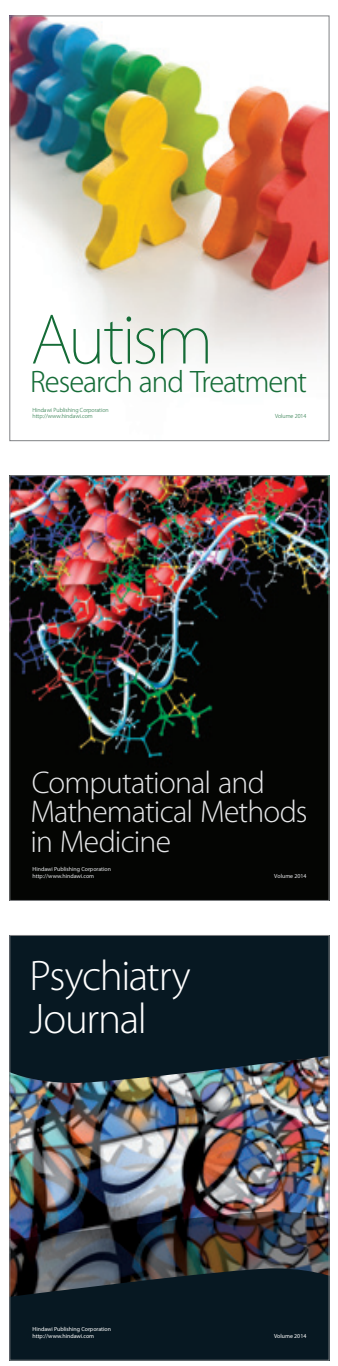
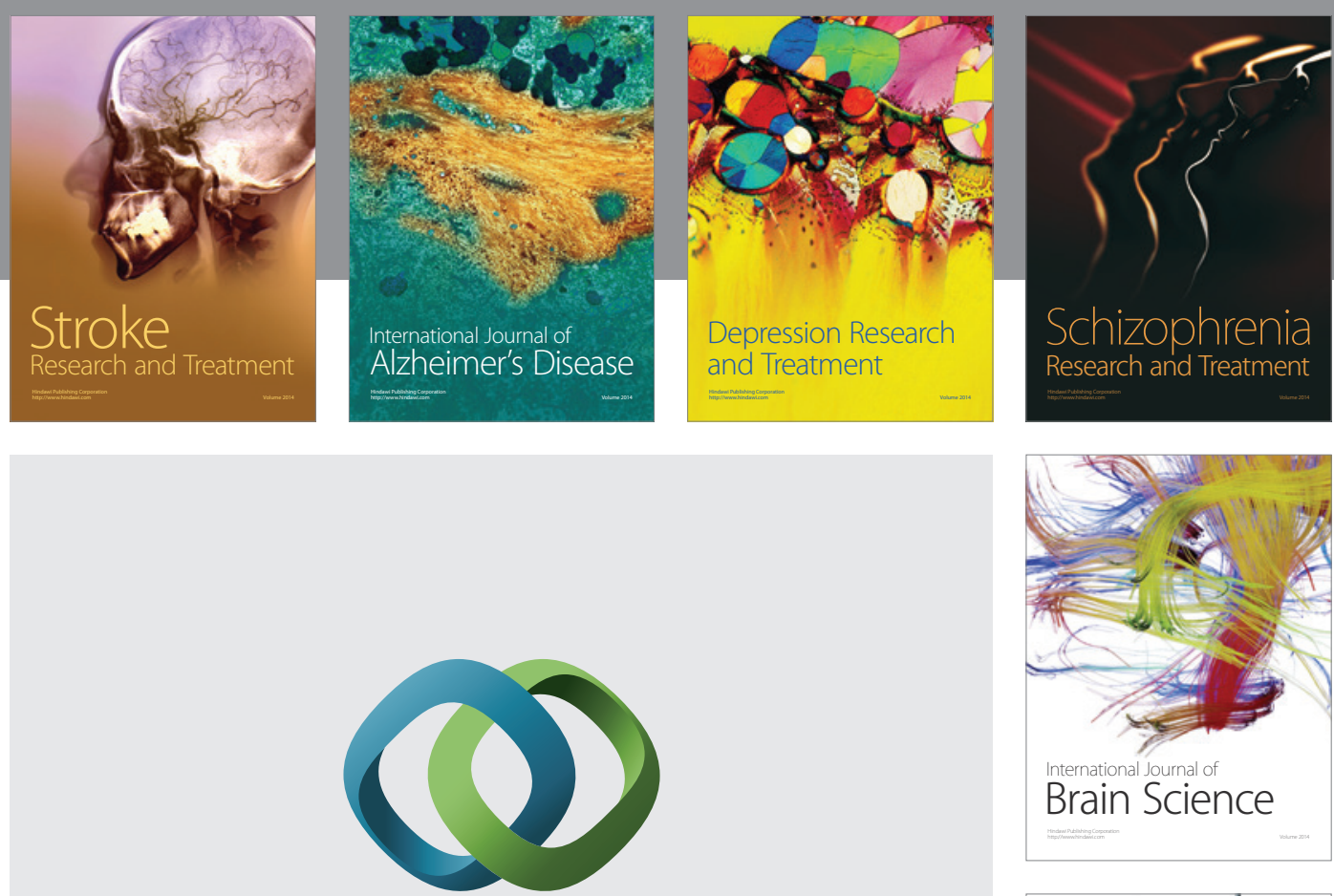

\section{Hindawi}

Submit your manuscripts at

http://www.hindawi.com
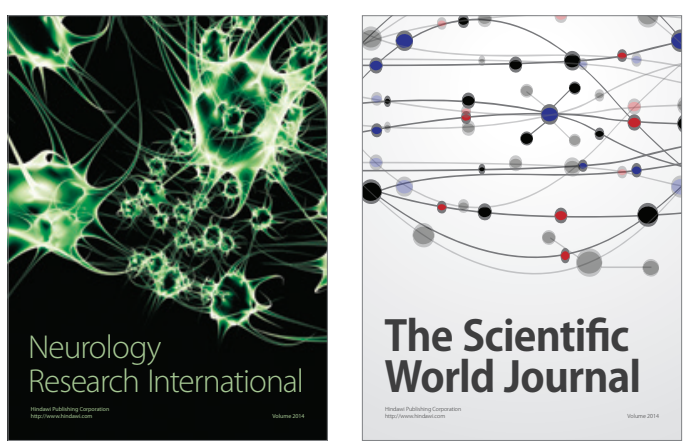

The Scientific World Journal

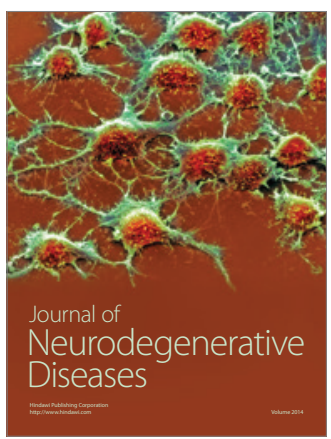

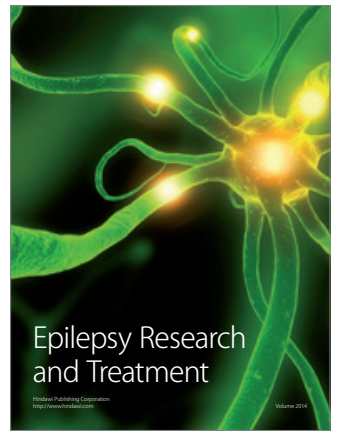

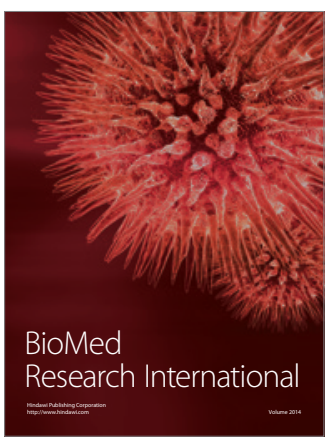

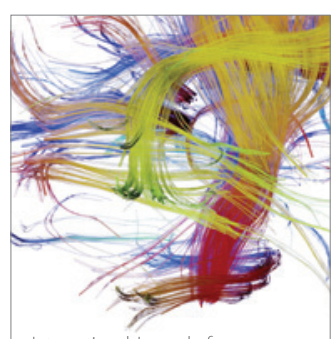

Brain Science

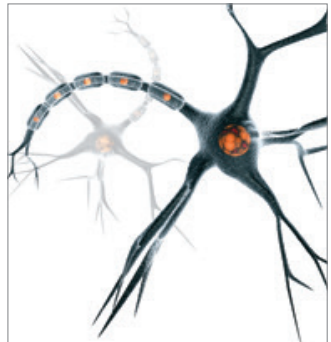

Neural Plasticity
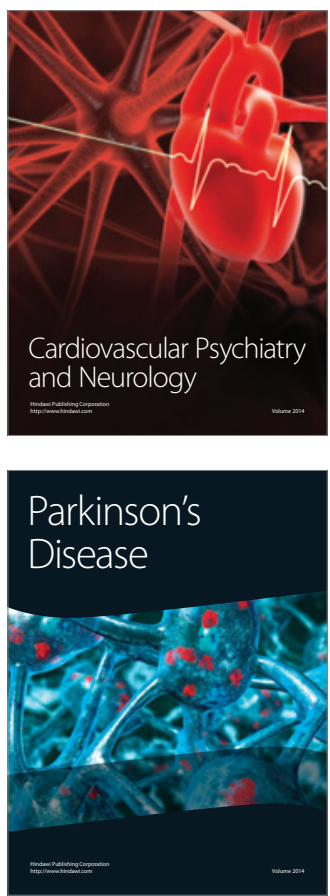